\title{
Distal versus proximal arm tremor in multiple sclerosis assessed by visually guided tracking tasks
}

\author{
Xuguang Liu, R Christopher Miall, Tipu Z Aziz, Jackie A Palace, John F Stein
}

\begin{abstract}
Objectives-To compare action tremor (AT) during manual tracking in normal subjects and patients with multiple sclerosis with tremor (MS-tremor group) and without tremor (MS-no tremor group), and to differentiate tremor occurring predominantly around the distal joint from that involving the proximal joints of the arm.
\end{abstract}

Methods-Subjects performed both a visually guided ramp tracking task using wrist flexion/extension and a whole arm circle tracking task using shoulder movement. Action tremor at the wrist or shoulder was computed as the SD of the tracking velocity. The ratio of wrist:arm tremor was then calculated to differentiate distal from proximal tremor in the tested arm. Frequency spectra of the records were also examined.

Results-During wrist tracking, AT in patients with multiple sclerosis contained a major frequency component at 4-5 Hz; the frequency was slightly lower during whole arm tracking. The ratio of wrist:arm tremor was significantly higher in the MS-tremor group. Of 12 tested arms, eight had tremor significantly weighted towards the distal joint, only one towards the proximal joint, and three had a ratio inside the control range.

Conclusions-AT in the arms of patients with multiple sclerosis can be effectively differentiated into proximal or distal using these two different tracking tasks. Despite the variability of the effects of multiple sclerosis, most of the AT was distal rather than proximal in this group of patients. Possibly conduction block along the corticocerebellocortical pathways caused this distal tremor.

(F Neurol Neurosurg Psychiatry 1999;66:43-47)

Keywords: tremor, multiple sclerosis, visual guidance, tracking, arm

Department of Clinica

Neurology, Radcliffe

Infirmary, Oxford

OX2 6HE, UK

$\mathrm{J}$ A Palace

Correspondence to:

Dr Xuguang Liu, University

Laboratory of Physiology,

Parks Road, Oxford OX1

3PT, UK. Telephone 0044

1865272116 ; fax 00441865

272469; email

xuguang.liu@physiol.ox.ac.uk

Received 5 March 1998 and in revised form 26 June 1998 Accepted 3 August 1998

Action tremor in the arm (AT, also called kinetic tremor, which presents during movement and may become worse on intention) is a major disabling symptom in patients with multiple sclerosis mainly due to inflammatory or demyelinating damage to cerebellar circuits in the CNS. ${ }^{1}$ The involuntary oscillations of AT superimposed on purposeful hand movements make the movements very difficult or even impossible to achieve. ${ }^{2}$ The AT in the arm not only distorts the controlled coordination of the shoulder, elbow, and wrist, but also upsets the stability or smoothness of the movement, both of which are required to perform even a simple goal directed reach-grasp task, such as reaching and grasping a cup of tea. Although such tremor presents as oscillation of the whole arm, in each subject the tremor tends to affect either the distal wrist joint or proximal shoulder joint more than the other, perhaps because the multiple sclerosis plaques disturb different somatotopic areas in the central motor system. Hence individual patients with multiple sclerosis may adopt different control strategies to compensate for whether the tremor affects the distal or proximal joints most, to obtain the best possible performance. The benefit from surgical alleviation of disabling tremor is related to tremor type and its distribution in various pathological conditions. ${ }^{3-6}$ The important point is that clinically it is found that thalamic stimulation or thalamotomy is less effective in alleviating proximal than distal tremor. ${ }^{5}$ Quantitative assessment of the magnitude and frequency of AT with differentiation of distal from proximal AT is, therefore, useful for planning surgical alleviation, for localising the damage in the central motor system, and for elucidating the control mechanisms compensating for AT.

Many natural tasks involve movement of the hand to visual targets. By contrast with rapid preprogrammed movements, control of precise and slow goal directed limb movements requires continuous on line guidance based on knowledge of the current positions of the guiding target and of the moving limb. Visually guided tracking tasks have thus proved useful in analysing motor control strategies ${ }^{7}$ and for investigating the relations between purposeful voluntary movements made to track a visual target and movement disorders, such as AT. ${ }^{2}$

We have therefore measured AT in the arms of patients with multiple sclerosis, differentiating distal from proximal AT. The magnitude and frequency of tremor, together with the underlying meaningful movement of either the wrist or shoulder, were separately analysed and qualitatively measured using two simple visually guided tracking tasks.
Subjects and methods

PATIENTS WITH MULTIPLE SCLEROSIS AND NORMAL CONTROLS

With local ethics committee approval, 11 patients with multiple sclerosis (seven men and four women) aged between 21 and 52, average 38 years, were tested. Patients were either outpatients at the Department of Neurology or inpatients at the Department of Neurosurgery, Radcliffe Infirmary, Oxford. All had a diagnosis 
of either clinically definite multiple sclerosis or laboratory supported definite multiple sclerosis according to the classification of Poser et al. ${ }^{8}$ They were also classified clinically as either relapsing-remitting multiple sclerosis $(n=5)$ or secondary progressive multiple sclerosis $(n=6)$. No patient had any complaint of severe disturbance of position sense in the upper limb. Before testing, eyesight was assessed by displaying both visual cues at a distance of $1 \mathrm{~m}$, and one patient with severe sight deficits was excluded from the study; all patients included reported that the display was easily seen. Also, the included patients had neither severe spontaneous nystagmus nor diplopia, which would interfere with the perception of the visual cues. Tracking performance of left and right arms was tested separately for each subject and the results were analysed in terms of the number of arms rather than people. Results from each arm were analysed separately, and it was established statistically that the arms behaved independently by showing that both sets of data had similar distributions (Kolmogorov-Smernov two variables test, $\mathrm{K}=0.30, \mathrm{p}>0.1)$, but were not correlated with each other $(r=0.38, \mathrm{p}>0.1$, $\mathrm{n}=11$ ).

Because their AT was the primary measure to be focused on, the patients were divided into two groups based on observation of AT just before the tracking tests: those with AT (MS-tremor, 12 arms in six patients) and those without AT (MS-no-tremor, 10 arms in five patients). Healthy subjects without neurological deficits (10 arms in five people, age range 17-40 years) formed the normal control group. Thus we had two different kinds of control group to compare with the MS-tremor groupnamely, the MS-no-tremor group, and normal subjects. It enabled us to compare the deficits caused by tremorogenic and non-tremorogenic lesions.

VISUALLY GUIDED WRIST RAMP TRACKING TASK Our pursuit wrist tracking task has been previously described in detail ${ }^{2}$ and is summarised as follows: a target consisting of a $12 \times 12$ pixel hollow square was displayed on a computer screen. It was initially stationary near one side of the screen; at the start of each trial, it moved horizontally at a constant speed to the other side of the screen, and then stopped. Target velocities were $13.64,9.23,7.50$, and $5.50 \%$, and were randomly allocated among 16 flexion movements giving four trials at each target velocity. The subject's forearm was supported in an adjustable plastic splint fixed to the arm of a chair, adjusted for each subject to firmly hold the forearm while allowing comfortable wrist flexion and extension over a range of $60^{\circ}$ $\left( \pm 30^{\circ}\right.$ around the neutral position) in the horizontal plane. The subject held a low resistance hand held joystick during wrist flexion and extension. The joystick position was displayed on screen as a $6 \times 6$ pixel hollow square cursor. The subject was instructed to make a wrist flexion movement to keep the cursor inside or as near to the moving target as possible, and then to move back to the starting position with an unpaced extension movement for the next trial.

VISUALLY GUIDED ARM CIRCLE TRACKING TASK As before, a $12 \times 12$ pixel hollow square target was displayed on the computer screen. It was initially stationary in the middle at the bottom of the screen; at the start of each trial, it moved in a $15 \mathrm{~cm}$ diameter circle, at a constant speed of one circle/10.2 s for four consecutive circles, and then stopped. The joystick position was displayed on screen as a $6 \times 6$ pixel hollow square cursor. The subject held a lightweight $70 \mathrm{~cm}$ long joystick and was asked to track the target by moving at the shoulder joint while keeping the elbow straight. The circular trajectory of the hand was thus about $40 \mathrm{~cm}$ in diameter.

TRACKING DATA ACQUISITION AND ANALYSIS The voltage signals generated by the joystick in each tracking task, proportional to the $x$ and $y$ position of the hand, was amplified and digitally sampled with 12 bit resolution at 70 Hz. Data were stored on a PC for further analysis.

The wrist and arm position signals were digitally differentiated and filtered using a zero phase, four pole, Butterworth filter (corner frequency $25 \mathrm{~Hz}$ ). A computer algorithm then selected tracking segments beginning 1 second after the target started moving until the end of the trial, thus eliminating the subjects' initial reaction delay and acceleration phases. In the arm tracking task, movement velocity in the $x$ and $y$ axes was combined as $\mathrm{dr} / \mathrm{dt}, \mathrm{r}=\sqrt{x^{2}+y^{2}}$. The computer then determined the target velocity, the mean movement velocity, and the SD of movement velocity (SD-MV), for each of the 16 trials in the wrist tracking task and for each of the 16 segments of $90^{\circ}$ of four circular movements in the arm tracking task. Means and SD over trials were then calculated for each tested arm.

The severity of AT was quantified by calculating the SD-MV; for perfect, smooth tracking the SD of the movement velocity would be zero. This measure is sensitive to the scatter of velocities around the mean tracking velocity, and is thus analogous to measurement by accelerometry. The frequency composition of the tracking records was also computed. The same segments of the velocity records were used as above, and the mean velocity was removed from each segment. The data were padded with zeros to provide 1024 data points/ segment and the Fourier transform calculated. Mean power spectra were calculated from the trials per subject, and averaged across patients in both the MS-no-tremor and the MS-tremor groups. A simple index of relative wrist:arm tremor score was calculated as the ratio of SD-MV in wrist tracking relative to that in arm tracking.

The accuracy of tracking was expressed as the percentage mean velocity (MV) of the subjects' movement relative to that of the target in both tasks; thus perfect tracking would have a value of $100 \%$. Impaired control of MV was 
Table 1 The wrist tracking task: comparison of tremor (SD-MV), error in movement velocity (EV), and simple reaction time (SRT) in normal controls and patients with multiple sclerosis (MS)

\begin{tabular}{llll}
\hline & $S D-M V(\%)$ & $E V(\%)$ & $S R T(s)$ \\
\hline Normal controls $(\mathrm{n}=10)$ & $65.77(13.28)$ & $5.57(1.52)$ & $0.26(0.02)$ \\
MS-no tremor $(\mathrm{n}=10)$ & $76.88(16.64)$ & $6.83(2.17)$ & $0.28(0.05)$ \\
MS-tremor $(\mathrm{n}=12)$ & $435.29(694.84)$ & $8.52(6.10)$ & $0.35(0.12)$ \\
Single factor ANOVA & $\mathrm{p}=0.009$ & $\mathrm{p}=0.43$ & $\mathrm{p}=0.024$ \\
\hline
\end{tabular}

Data are presented as means (SD).

reflected in the absolute percentage error in the movement velocity $(\mathrm{EV})$ relative to the target velocity.

MEASUREMENT OF VISUALLY CUED SIMPLE REACTION TIME

Simple reaction time (SRT) movements of the wrist were recorded after a visual cue given by a white square (now $20 \times 20$ pixels in size) jumping from side to side on the screen at random intervals of 2-4 seconds. Subjects were instructed to move the joystick as fast as possible by wrist flexion or extension when the target jumped, without worrying about the accuracy of movement. No visual feedback of wrist movement was displayed. Mean SRT were calculated as the time difference between when the target and subjects' movements started, averaged over six trials.

\section{Results}

WRIST TRACKING TASK IN NORMAL CONTROLS AND PATIENTS WITH MULTIPLE SCLEROSIS

There were no significant differences in wrist tracking SD-MV, EV, and SRT between the MS-no tremor group and the normal controls. As expected, the wrist SD-MV, our index of tremor, was significantly higher in the patients with AT than in the other two groups (table 1). Similarly, the wrist SRT was significantly prolonged in the MS-tremor group. There were no significant differences in $\mathrm{EV}$ among the three groups.

VISUALLY GUIDED ARM TRACKING TASK IN NORMAL CONTROLS AND PATIENTS WITH MULTIPLE SCLEROSIS

There were no significant differences in the whole arm SD-MV and EV between the MS-no tremor group and the normal controls.

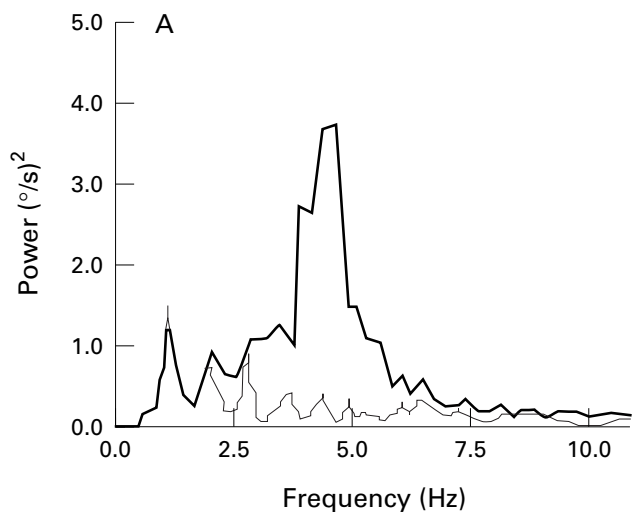

Table 2 The arm tracking task: comparison of tremor $(S D-M V)$ and error in movement velocity (EV) in normal controls and patients with multiple sclerosis (MS)

\begin{tabular}{lll}
\hline & $S D-M V(\% / s)$ & $E V(\%)$ \\
\hline Normal controls $(\mathrm{n}=10)$ & $20.68(3.20)$ & $12.43(12.49)$ \\
MS-notremor $(\mathrm{n}=10)$ & $25.39(7.32)$ & $14.74(20.06)$ \\
MS-tremor $(\mathrm{n}=12)$ & $117.99(126.33)$ & $23.69(27.01)$ \\
Single factor ANOVA & $\mathrm{p}=0.009$ & $\mathrm{p}=0.43$ \\
\hline
\end{tabular}

Data are presented as mean (SD).

As expected, arm SD-MV in the patients with AT was about four times as high as that in the other two groups (table 2).

CORRELATION OF THE TWO TRACKING TASKS AND DIFFERENTIATION OF THE DISTAL AND PROXIMAL AT

No significant correlation was found between the magnitude of AT assessed with wrist and arm tracking tasks $(r=0.39, \mathrm{p}=0.20)$. Examination of the averaged power spectra of the tracking velocity disclosed that the multiple sclerosis tremor during the wrist tracking consisted of a major component ranging between $2-7 \mathrm{~Hz}$, peaking at $4-5 \mathrm{~Hz}$. During the arm tracking the spectra were similar, but with a slight shift to a lower frequency of about $3.5 \mathrm{~Hz}$ (fig 1). There was no significant correlation in peak frequency of the tremor between two tracking tasks $(r=0.41, \mathrm{p}=0.18)$. A subpeak at about 1 $\mathrm{Hz}$ appeared in the spectra from all three groups due to intermittent visually guided corrections of position. ${ }^{2}$

Values of the calculated wrist:arm ratio of SD-MV in the three test groups were compared and are illustrated in fig 2 . There was no significant difference between the values for normal controls (3.18 (SEM 0.45)) and the MS-no tremor group (3.15 (SEM 0.76)). In comparison, the ratio was significantly increased in the MS-tremor group (6.51 (SEM 5.06), $\mathrm{p}=0.025)$. Further analysis was carried out by plotting the wrist:arm ratio of each tested arm (fig 3), comparing with the MS-notremor group. The 12 tested arms were unevenly distributed $\left(\mathrm{p}=0.039, \chi^{2}\right.$ test $)$ : eight arms had a wrist:arm ratio higher than the range of control group (mean value $\pm 1 \mathrm{SD}$ ); only one was lower; three arms were inside the control range.

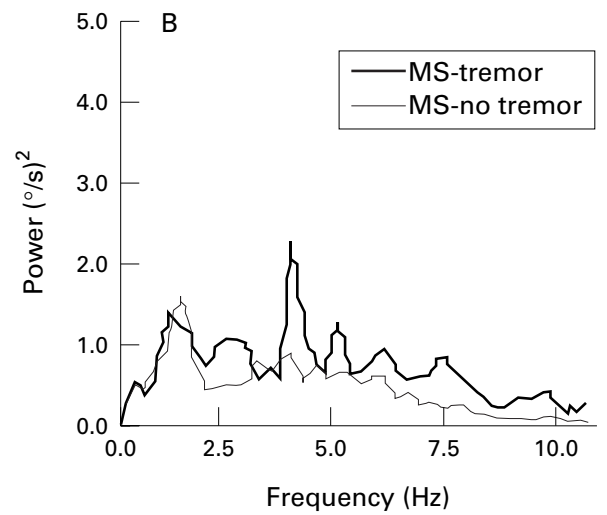

Figure 1 The mean power spectra of the MS-no-tremor group $(n=10)$ and the MS-tremor group ( $n=12)$ from the wrist tracking $(A)$ and arm tracking tasks $(B)$. Tremor shown by the two separated tracking tasks peaks at a similar frequency range of 4-5 Hz, with a slight shift to lower frequency in shoulder compared to wrist tremor. There are also subpeaks at about $1 \mathrm{~Hz}$ due to intermittent visually guided corrections of position. 


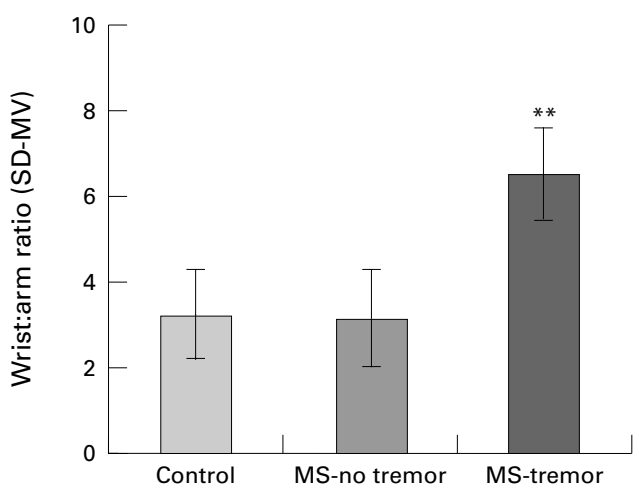

Figure 2 Comparison of the wrist:arm ratio of SD-MV among three tested groups of the normal control $(n=10)$, $M S$-no-tremor $(n=10)$ and $M S$-tremor $(n=12)$. Results are means (SEM). ${ }^{\star \star} p=0.025$, single factor ANOVA.

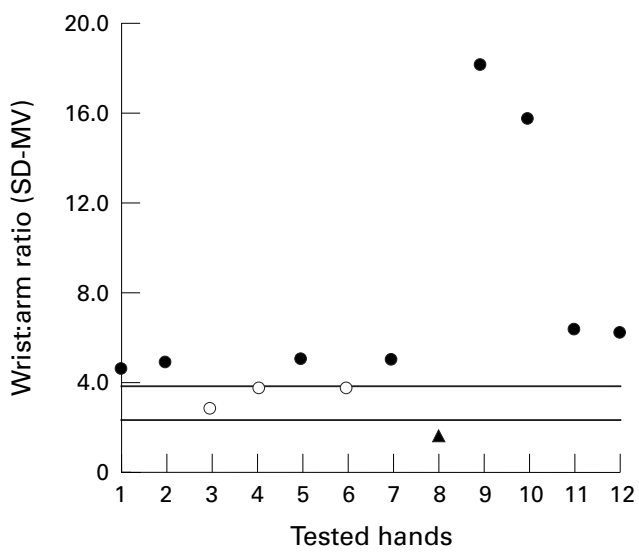

Figure 3 Distal tremor $(\bullet)$ was detected in eight tested arms within the MS-tremor group indicated by wrist:arm ratios above the control range; the horizontal lines indicate $+/-1 S D$ about the mean ratio of the control group.

Proximal tremor (A) was seen in only one arm with its wrist:arm ratio below the control range. Tremor in the remaining three arms tested within the MS-tremor group (O) lay within the control range.

\section{Discussion}

The variable nature of multiple sclerosis - in terms of the size, number, location, and pathogenic heterogeneity of lesions - produced great differences between the patients we studied. This raises difficulties in interpreting our results when compared with those from patients with more clearly localised lesions. We have tried to minimise these difficulties by restricting our observations to tremor and tracking movements measured within the visually guided tracking tasks that we have applied; we cannot yet extrapolate from these simple tracking tasks to more natural visually guided movements.

We studied the relations between AT, accuracy of tracking velocity, and SRT in patients with multiple sclerosis in an earlier publication. ${ }^{2}$ We found that the severity of AT was independent of delayed SRT, and independent of the accuracy of tracking velocity. However, we found that SRT did correlate with errors in tracking velocity (EV). The prolongation of SRT and the EV indicate that delayed initiation and impairment in control of movement velocity coexisted in these patients. More interestingly, the prolongation of SRT significantly correlated with the magnitude of $\mathrm{EV}$, which suggests that control of both initiation and velocity of a movement may share a common segment of the motor control circuit. One obvious candidate to explain these correlations is the corticocerebellocortical loop, which is often affected in multiple sclerosis. Motor commands are projected from the motor cortex via the corticopontocerebellar pathway to the cerebellum, and return via the cerebellothalamocortical pathway back to the motor cortex. This may explain how multiple sclerosis lesions in different anatomical locations could result in impairment in this pathway and therefore a similar clinical picture of AT. Evidence suggests that this motor control loop is responsible for aspects of the planning, initiation, and execution of movement, including the maintenance of constant velocity and force. ${ }^{9} 10$

To distinguish a tremor component contributed by one particular joint, a test has to be selective. During the wrist tracking task, the AT of the wrist was well isolated as both the shoulder and elbow were abducted while the subject sat with the forearm secured to the chair. In the arm tracking task, the elbow was extended and the wrist was held fixed by the subject, so that the movement was mainly executed by the shoulder. It has to be pointed out, however, that these two tracking tasks were different: one was a linear ramp for the wrist tracking and the other was a two dimensional circular ramp for the arm tracking task. Therefore, a simple comparison between the values of SD-MV obtained from these two tasks is inappropriate to decide whether wrist tremor was more severe than shoulder tremor. This conclusion came from comparing the ratio between the wrist and arm tremor scores in the MS-tremor group with those in the MS-no tremor group and normal controls. Thus we consider only the relative change in this ratio between the groups, so any intrinsic differences between two tracking tasks were largely cancelled out.

Perhaps the most intriguing result was that the ATs recorded in each task peaked at a similar frequency range of $4-5 \mathrm{~Hz}$, but with no correlation in either magnitude or peak frequency. This suggests that ATs, whether predominantly from distal or proximal arm joints, may originate from a similar pathological origin in the central motor system, possibly the cerebellar loop. ${ }^{2}$ Hence the tremors at different joints were probably not completely independent. Nevertheless, the contribution of each joint to the magnitude of the overall tremor was unevenly distributed among the arm segments. It might be anticipated that tremor measured by a hand held joystick would be greater during movements involving the whole arm. The overall tremor measure would be contributed to by each joint, as wrist tremor would add to that of the shoulder; alternatively equal angular motion of wrist and shoulder would be magnified by the long leverage of the arm. However, it was evident from a previous study ${ }^{11}$ and from the present results that the mechanical properties of the extended arm actually had a damping effect which resulted in smaller proximal than distal joint tremor. Also, the SD-MV wrist:arm ratio expresses the relative importance of distal 
or proximal tremor, rather than the overall severity of the AT. One arm can have a milder tremor, but show a more extreme ratio of wrist to shoulder tremor than another arm with more severe tremor of both joints.

We found in most of these patients with multiple sclerosis that visually guided whole arm movements, similar to everyday tasks such as reaching, were affected more by proximal than distal tremor, whereas the forearm and hand movement, related perhaps to tasks such as writing, were more affected by distal than proximal tremor. In normal situations, the arm has more degrees of freedom available than necessary to perform most simple movements, ${ }^{12}$ and therefore, the selective redundant freedom of the arm allows the patients to gradually develop a compensating control strategy as their AT develops. Such control is usually achieved by constraining the freedom of the disordered joint and maximising that of others. On the other hand, if a joint with less tremor is constrained, the overall movement of the arm becomes much worse. Some patients have complained that the control of their arms became less effective immediately after abolishing the action tremor by thalamotomy. ${ }^{2}$ Possibly they were not able to recall the normal control mechanism after having had tremor for a long time, but still used the control strategy which they had developed to compensate for the tremor but which was unsuitable when the tremor had been eliminated.

Given the variable nature of the AT among patients with multiple sclerosis, assessment that only measures the magnitude and frequency of the AT would be inadequate. With our simple visual guided tracking tasks, extra dimensions of the AT, such as the effect of visual feedback on the intention tremor, interaction of AT with intended movement, and differentiation of distal from proximal tremor can be disclosed. This information can be useful for understanding the pathophysiological mechanisms of tremor generation and for functionally localising the damage within the central motor system responsible for the tremor. It has been shown in preliminary results that the AT in multiple sclerosis can be suppressed by either electrical deep brain stimulation $^{513}$ or coagulation in the thalamus. ${ }^{2}$ However, clear criteria based on the adequate assessment of the AT for selecting appropriate treatment, determining the best target among the thalamic nuclei, and for evaluating effectiveness of the treatment will require comparative studies of multiple sclerosis tremor at preoperative and postoperative stages with a relative large population of patients. The visually guided tracking tasks presented here can be useful for this purpose.

We are very grateful to the patients and normal subjects for their cooperation, and to Miss Helen Miles for her help with recruiting patients with multiple sclerosis. We also thank the McDonnell-Pew and MRC centres for Cognitive Neuroscience, Oxford. RCM and XL are supported by the Wellcome Trust.

1 Tranchant C, Bhatia KP, Marsden CD. Movement disorders in multiple sclerosis. Mov Disord 1995;10:418-23.

2 Liu X, Miall RC, Aziz TZ, et al. Analysis of action tremor and impaired control of movement velocity in multiple and impaired control of movement velocity in multiple Disord 1997;12:992-9.

3 Hubble JP, Busenbark KL, Wilkinson S, et al. Effects of thalamic deep brain stimulation based on tremor type and diagnosis. Mov Disord 1997;12:337-41.

4 Benabid L, Pollak P, Gervason C, et al. Control of tremor by chronic stimulation of the ventral intermediate thalamic nucleus. Lancet 1991;337:403-6.

5 Nguyen JP, Degos JD. Thalamic stimulation and proximal tremor. Arch Neurol 1993;50:498-500.

6 Hirai T, Miyazaki M, Nakayima $\mathrm{H}$, et al. The correlation between tremor characteristics and the predicted volume of effective lesions in stereotaxic VIM thalamotomy. Brain 1983;106:1001-18.

7 Miall RC, Weir DJ, Stein JF. Intermittent responses in human manual tracking. F Motor Behav 1993;25:53-63.

8 Poser CM, Paty DW, Scheinberg L, et al. New diagnostic Poser CM, Paty DW, Scheinberg L, et al. New diagnostic
criteria for multiple sclerosis. Guidelines for research criteria for multiple sclerosis. Guideline
protocols. Ann Neurol 1983;13:227-31.

9 Stein JF. Role of the cerebellum in the visual guidance if Stein JF. Role of the cerebellum in the
movement. Nature 1986;232:217-21.

10 Horne MK, Butler EG. The role of the cerebello-thalamocortical pathway in skilled movement. Prog Neurobiol 1995; 46:199-213.

11 Hore J, Wild B, Diener H-C. Cerebellar dysmetria at the elbow, wrist, and fingers. $\mathcal{F}$ Neurophysiol 1991;65:563-71.

12 Bernstein N. The coordination and regulation of movement. London: Pergamon 1967.

13 Brice J, McLellan L. Suppression of intention tremor by contingent deep-brain stimulation. Lancet 1980;i:1221-2. 